\title{
Diagnóstico del uso de las TIC en la educación de la UNAH-TEC Danlí, 2014
}

Pedro Martín Fiallos Gonzales ${ }^{1}$

Jacobo Paredes Heller ${ }^{2}$

\section{RESUMEN}

La presente investigación se realizó en el centro universitario UNAH-TEC Danlí en el departamento de El Paraíso, en el cual parte de su visión es la educación con orientación tecnológica. Se consideró para esta investigación como objetivo general, diagnosticar el uso de las tecnológicas de la información y la comunicación (TIC), mediante un análisis de las condiciones del contexto educativo universitario para el tercer periodo académico del año 2014, cuyas dimensiones con relación a estas tecnologías son los roles del profesor y estudiantes, satisfacción estudiantil, capacitación docente y estado de los recurso tecnológicos.

El enfoque de investigación es de tipo mixto (cuali-cuantitativo) combinando: lo cuantitativo que manipula datos porcentuales, anclado a la técnica de la observación, el cual suma el carácter cualitativo enfocado en el área tecnológica. Los instrumentos usados son de tipo encuesta, que fueron aplicadas a 44 profesores y 274 estudiantes, incluyendo la muestra elementos de las tres especialidades ofertadas en el centro. Por lo anterior y como característica del método mixto, se realizó la observación directa de los recursos tecnológicos existentes como apoyo a la investigación, cuyo propósito es la descripción de las condiciones actuales en las que se encuentran, describiendo sus características técnicas.

Se puede considerar que el resultado obtenido muestra que existe un desaprovechamiento de los recursos que brindan las TIC, lo que refleja una baja frecuencia de uso con $72.03 \%$ y $74.64 \%$ para estudiantes y profesores respectivamente. También se observa un nivel bajo de competencias de parte de los profesores, con un $58.96 \%$

${ }^{1}$ Beneficiario de una beca docente egresado de posgrado de la DICYP, Departamento de Informática Administrativa, UNAH-TEC Danlí: pedroeneva@gmail.com

${ }^{2}$ Asesor, Facultad de Posgrado, UNITEC: jacobo.paredes.heller@unitec.edu 
percibido por los estudiantes, así como bajo apoyo al proceso de enseñanza-aprendizaje; no obstante, se muestra un porcentaje del $73 \%$ aceptable de actitud positiva ante el uso de nuevas tecnologías educativas por parte de las poblaciones estudiadas. Aunado al análisis de la investigación se definió la perspectiva del uso de las $\mathrm{TIC}$, identificando un bajo porcentaje en cuanto a su aplicabilidad en el contexto universitario, ya que las asignaciones ligadas a estas es de $55.33 \%$. Se busca con dichos resultados la base fundamental para el establecimiento de recomendaciones orientadas a superar las debilidades encontradas en el uso de la TIC, proporcionando a las autoridades un plan de acción como punto de apoyo orientado a la toma de decisiones. Se espera, además, despertar con este artículo el interés de las autoridades locales y de la ciudad universitaria.

Palabras clave: competencias, diagnosticar, TIC, tecnología educativa.

\section{ABSTRACT}

This research was conducted at the UNAH-TEC Danlí university campus, in the department of El Paraíso; where one of the vision's dimensions is technology-oriented education. The following research considered a general objective defined as: Use of Technology Information and Communication Technologies (ICT) Diagnosis. The conditions of the educational context were analyzed, during the year of 2014. The dimensions of these technologies are related to each of the roles of teachers and students, student satisfaction, teacher training and state of the technology resources.

The focus of research is a mixed type combining qualitative and quantitative approaches: quantitative data manipulating percentages, based on the observation technique which adds the qualitative nature to the technology area. The survey instruments used were applied to 44 teachers and 274 students including the three specialties offered in the student center. As a result of using a mixed method, the existing technological resources were directly observed as research support, in order to define their conditions and consider their characteristics as techniques. The obtained results showed that there is a misuse of ICT resources, reflecting a low frequency of use of $72.03 \%$ by students and $74.64 \%$ by teachers, students' perception on teachers' competences as low as $58.96 \%$ in addition to a low support for the teaching-learning process. However, an acceptable positive attitude towards the use of new educational technologies was shown (73\%). 
The perspective of ICT use was defined by identifying a low percentage in terms of their applicability to the university context. (55.33\%). These results search for an establishment of recommendations to overcome the weaknesses found in the use of ICT, in order to define an action plan for the authorities. In addition, this article hopes to awaken the interest of the university authorities and university campuses nationwide.

Keywords: competencies, diagnose, TIC, educational technology. 


\section{INTRODUCCIÓN}

Las tecnologías de la información y comunicación en educación se refieren al: "Uso de recursos tecnológicos hardware, software e infraestructura tecnológica para mejorar el proceso de enseñanza y aprendizaje" (Saravia y Eguigure, 2002). Por consiguiente, esta investigación se concentra en el contexto educativo del centro universitario UNAH-TEC Danlí, teniendo como entidades de estudio a los profesores y estudiantes, buscando medir las dimensiones tales como los roles (papeles) del profesor y estudiante, satisfacción estudiantil, capacitación docente y estado de los recursos tecnológicos; visualizándose estas como variables independientes; la variable porcentajes de uso como variable dependiente y la última, referente al estado de los recursos, será definida como una descripción de su condición actual.

Como raíz de la investigación se ha establecido una pregunta genérica aplicada a una muestra de 149 estudiantes y 21 profesores, orientada a detectar bajo su criterio cuál es el aprovechamiento en el uso y manejo de la TIC. Como respuesta se obtuvo un porcentaje negativo del $20.13 \%$, siendo este el problema encontrado, el cual se debe superar.

Todo esto lleva al planteamiento de un diagnóstico, el cual permite definir las variables dependientes e independientes en el estudio, de las cuales extraeremos información para establecer conclusiones y respuestas a nuestros objetivos y preguntas de investigación. Es preciso mencionar que todas las debilidades encontradas se abordarán proponiendo un plan de acción que sirva de apoyo para la toma de decisiones, permitiendo el fortalecimiento del uso de las TIC en el contexto de la UNAH-TEC Danlí.

Dentro de este contexto universitario la investigación percibe hallazgos que serán parte de la respuesta para el problema planteado. Asimismo, ofrece beneficios directos hacia las entidades involucradas del centro universitario, además se vuelve necesario por la situación actual del centro sobre el uso de las TIC como recurso tecnológico, siendo base para la identificación de fortalezas y debilidades con relación a dimensiones como roles, satisfacción, capacitaciones y estado del recurso tecnológico.

Se busca con los resultados enfocarse en acciones para reforzar las debilidades sirviendo de fuente para el planteamiento de innovaciones metodológicas a nivel de aula y se espera que dicha investigación beneficie a profesores, estudiantes, 
autoridades locales y administrativas, ya que aporta un panorama de la realidad universitaria en el uso de las tecnologías de la información y la comunicación.

Cabe mencionar que la investigación se realizó en un periodo de 6 meses, enfocada en profesores y estudiantes, buscando la obtención de la información de todas las variables mediante la aplicación de instrumentos estructurados, tipo encuesta, enriquecidos con la observación, la cual ayuda a lograr uno de los objetivos de investigación.

Se busca reconocer la importancia de mejorar las competencias del uso de las TIC en el contexto, mejorando así los estándares de calidad en la educación del centro; otro beneficio directo es el apoyo al proceso de enseñanza y aprendizaje. Además, el plan de acción resultante intentará que las autoridades tomen conciencia sobre la necesidad de desarrollar acciones que busquen volver eficiente el uso de las TIC.

Se espera que la investigación resumida en este artículo tenga un impacto positivo en la gestión educativa y administrativa del centro y que genere el interés de las autoridades centrales de la UNAH (ciudad universitaria) para impulsar y mejorar el uso de las TIC en los procesos académicos y demás centros regionales.

\section{METODOLOGÍA}

Se ha definido un enfoque mixto, combinando características cuantitativas con cualitativas, permitiendo el primero el conteo de datos para procesarlos como elementos porcentuales (Gómez, 2006); caracterizando lo cualitativo está la técnica de la observación enfocada en el área tecnológica; igualmente el carácter de diseño no experimental, ya que no se manipulan las variables, pero sí se toma su contenido e información según cómo se encuentra en el contexto universitario en estudio.

En el caso de la observación de los factores tecnológicos, se inició con una revisión del inventario del equipo existente, procediendo a visitar cada departamento que forma parte de la estructura administrativa para verificar la cantidad, condiciones y estado de dicho equipo.

Se tomó nota de la configuración básica de las computadoras de cada laboratorio siguiendo los pasos técnicos para su verificación directa, se observaron con detalle las condiciones externas del equipo, así como de las condiciones pedagógicas de las 
aulas y laboratorios y de las conexiones existentes de infraestructura de conectividad. Posteriormente, se elaboró un resumen detallado que permitiera definir las condiciones de los recursos y del contexto pedagógico. Es preciso mencionar que la recopilación de la información se realizó en el segundo periodo y tercer académico comprendido del 9 de junio al 30 de octubre de 2014, por lo que el estudio es de tipo transversal, recopilando los datos en un momento académico único.

Para una mejor comprensión de los resultados, se ha considerado un análisis sobre las teorías de aprendizaje existentes, relacionándolo con roles de los profesores y alumnos en los procesos educativos vinculándolo con el uso de las TIC, lo que permite comprender mejor cuál ha sido la evolución de los papeles de los entes involucrados en el proceso educativo, permitiendo un apoyo inductivo para el análisis de datos (Gómez, 2006).

Se ha delimitado la población con la que se ha trabajado, definiéndose esta como la: "Totalidad de un fenómeno de estudio o entidades de la población que integran dicho fenómeno" (Tamayo, 2001, p.176). Asimismo, se han extraído dos muestras: profesores y estudiantes, volviéndose entidades claves del estudio, siendo la base para determinar las características de las variables identificadas en esta investigación.

La muestra poblacional se delimitó para todos los estudiantes de las tres especialidades en todas sus jornadas (días de semana y sabatinas), buscando que sea heterogéneo, incluye estudiantes temporales matriculados en otras carreras que no oferta la UNAH-TEC Danlí, pero que estudian sus clases generales en este centro. Otra población delimitada es la totalidad de los profesores que de igual forma corresponden a las tres especialidades, siendo un total de 44 seleccionados de acuerdo a la fórmula matemática; según Sampieri (2006), planteada en los párrafos siguientes.

Conociendo el tamaño de nuestros estratos de población y definiendo el nivel de confianza en un $95 \%$ equivalente al 1.96 de coeficiente, el error de la muestra de 5 $\%$ y las probabilidades de éxito y fracaso al $50 \%$, en donde:

$\mathrm{N}=$ tamaño de la población

$Z$ = nivel de confianza (coeficiente de 1.96)

$\mathrm{P}=$ probabilidad de éxito $(50 \%)$

$\mathrm{Q}=$ probabilidad de fracaso $(50 \%)$

$\mathrm{D}=$ precisión 0 error para la proporción (5\%) 
De esta manera el cálculo de la muestra para cada población (profesores, estudiantes) generó como resultado:

1. Profesores con población de 49, en donde:

$$
n=\frac{49 \times 1.96^{2} \times 0.5 \times 0.5}{0.05^{2} \times(49-1)+1.962 \times 0.5 \times 0.5}
$$

el tamaño de la muestra para docentes es de: 44.

2. Los estudiantes de las carreras con población de 950, en donde:

$$
n=\frac{950 \times 1.96^{2} \times 0.5 \times 0.5}{0.05^{2} \times(950-1)+1.962 \times 0.5 \times 0.5}
$$

el tamaño de la muestra para esta población es de: 274.

Utilizando el muestreo por cuotas se dividió el total de la muestra entre el porcentaje de cada carrera, cuyos resultados se muestran en la tabla 1.

Tabla 1. Cálculo de muestras de la población de estudiantes

$\begin{array}{lccc}\begin{array}{l}\text { Facultades del } \\ \text { estrato de } \\ \text { estudiantes }\end{array} & \begin{array}{l}\text { Cantidad de } \\ \text { estudiantes en } \\ \text { cada facultad }\end{array} & \begin{array}{l}\text { Cantidad de muestra } \\ \text { representativa al porcentaje } \\ \text { de estudiantes }\end{array} & \begin{array}{l}\text { Porcentaje de estudiantes } \\ \text { por facultad con relación al } \\ \text { total de la población }\end{array} \\ \begin{array}{l}\text { Licenciatura } \\ \text { en Enfermería }\end{array} & 387 & 112 & 40.74 \% \\ \begin{array}{l}\text { Ingeniería } \\ \text { Agroindustrial }\end{array} & 87 & 25 & 9.16 \% \\ \begin{array}{l}\text { Licenciatura } \\ \text { en Informática }\end{array} & 214 & 62 & 22.53 \% \\ \text { Estudiantes de } & & 76 & 27.58 \% \\ \text { facultades temporales } & 262 & 274 & 100 \% \\ \text { Totales } & 950 & 76 & \end{array}$

Fuente: Registro UNAH-TEC Danlí, 2014. 
Tabla 2. Cálculo de la muestra de población de profesores

$\begin{array}{lccc}\begin{array}{l}\text { Facultades del } \\ \text { estrato de } \\ \text { profesores }\end{array} & \begin{array}{l}\text { Cantidad de } \\ \text { profesores en } \\ \text { cada facultad }\end{array} & \begin{array}{l}\text { Cantidad de muestra } \\ \text { representativa al porcentaje } \\ \text { de estudiantes }\end{array} & \begin{array}{l}\text { Porcentaje de estudiantes } \\ \text { por facultad con relación al } \\ \text { total de la población }\end{array} \\ \begin{array}{l}\text { Licenciatura } \\ \text { en Enfermería }\end{array} & 22 & 44.90 \% & 20 \\ \begin{array}{l}\text { Ingeniería } \\ \text { Agroindustrial }\end{array} & 15 & 30.61 \% & 13 \\ \text { Licenciatura } \\ \text { en Informática }\end{array}$

Fuente: Registro UNAH-TEC Danlí, 2014.

En los párrafos anteriores ha sido importante definir cuál es la unidad de análisis con la que se ha trabajado, entendiendo esta como "el objeto de estudio, que comúnmente son individuos, sin embargo, pueden ser grupos" (Vivanco, 2005).

En el aspecto cuantitativo para el procesamiento de las encuestas se usó el software SPSS, apoyado por el software Microsoft Excel 2010, tanto para sumas y cálculos de porcentajes. Es importante aclarar que solo cuando se describe la existencia del equipo tecnológico se usa una descripción cualitativa del recurso TIC, definiéndose las características técnicas y de actualización del hardware y del software, ancho de banda de la red internet anclado al uso de la plataforma virtual Moodle, así como las condiciones en que se encuentran los equipos tecnológicos de los laboratorios, como en los diferentes departamentos de la estructura organizativa institucional local.

\section{RESULTADOS Y ANÁLISIS}

En la encuesta aplicada a las muestras poblacionales en el contexto universitario, con relación a las preguntas de investigación, se definió una escala de medición que va del uno hasta el cinco, siendo cinco el de mayor aceptación, todos los porcentajes que a continuación se definen en cada gráfico usan esta escala estadística y porcentual. 


\section{Percepción de estudiantes y profesores}

Para poder hablar del porcentaje del uso de las TIC reflejada como variable dependiente en este estudio diagnóstico, se ha definido una gráfica que determina cuáles son las actividades de uso frecuente en el uso del internet, permitiendo visualizar cuál es la iniciativa de los docentes y alumnos en la potenciación de este recurso.

Si se definen las actividades de uso frecuente de las TIC en el contexto, se presenta una síntesis de datos de las tres especialidades de las muestra profesores y estudiantes.

Figura 1. Actividades más frecuentes en el uso del internet

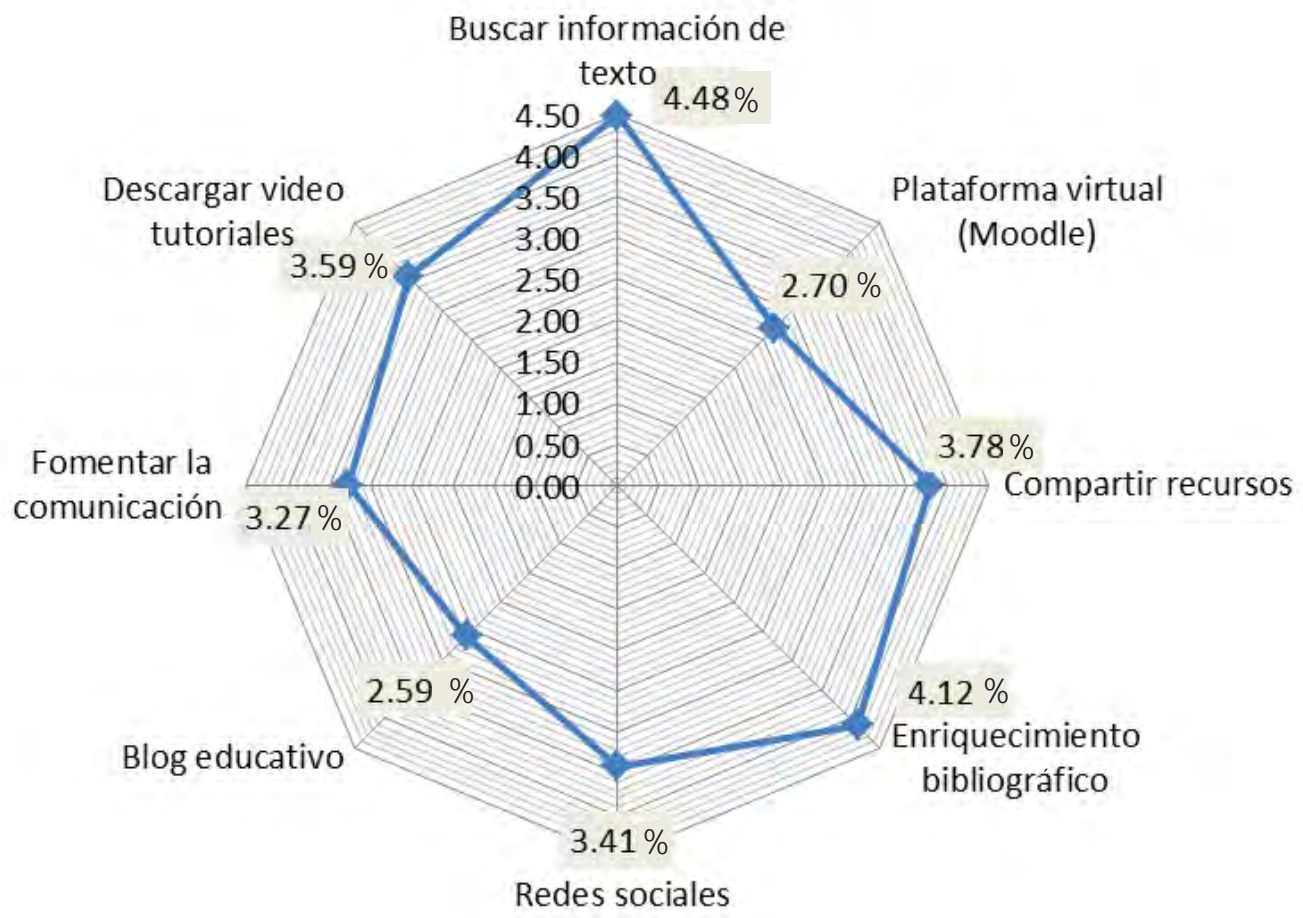

La figura 1 refleja diferentes opciones para maximizar el uso de las TIC y de acuerdo a su uso se define en porcentajes, se analiza que el recurso poco usado es la plataforma Moodle. También se observa el poco uso de los blog educativos, siendo este último un recurso gratis que ofrece la internet, los porcentajes coinciden con la observación de que hay poco interés de gestión por parte de profesores y autoridades 
en incorporarlo a los planes de clase para maximizar su uso en el desarrollo de contenidos curriculares propios de cada asignatura.

Pero, fuera de las aulas, el uso de las TIC se evidencia por la descarga de video tutorial y enriquecimiento bibliográfico, recalcando que las redes sociales de igual manera representan un porcentaje de alto uso, reflejando aquí un interés de los poblaciones estudiadas en el uso de las TIC en el contexto educativo, al menos en los aspectos de fomento de la comunicación e intercambio de información.

Si nos referimos a la frecuencia de uso de las TIC en la UNAH-TEC Danlí, se refleja un análisis cada uno de estos recursos en la figura 2.

Figura 2. Frecuencia de uso de los recursos TIC existentes en el centro

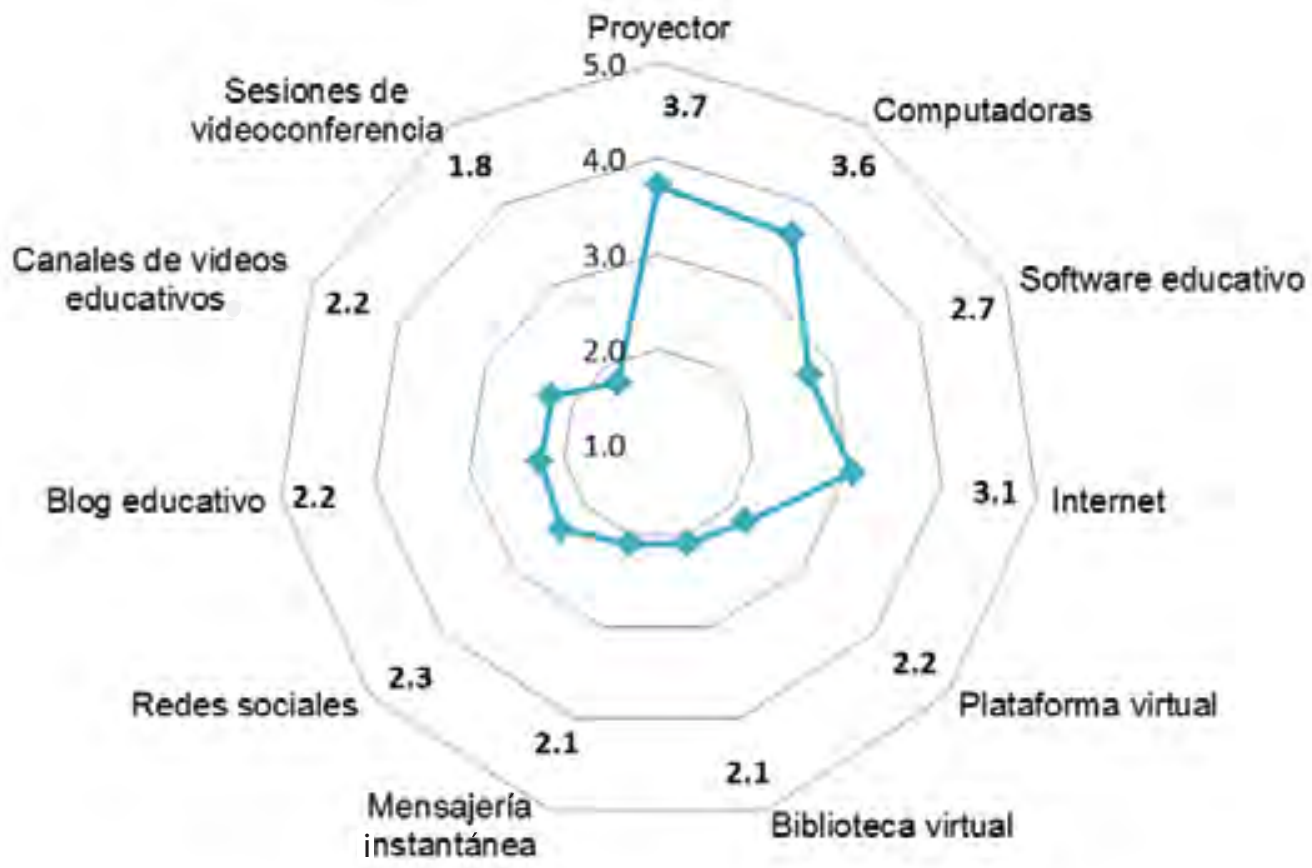


La figura 2 anterior define en su generalidad porcentajes bajos y preocupantes, por ejemplo, uso de los recursos y la videoconferencia, es de menor uso anclado a su escaso ancho de banda en la internet, lo que significa una limitante clave para la academia dentro y fuera de las aulas, pero lo que motiva bastante es el uso de la computadora, celulares y PDA con conexión a internet con recurso personal propio, lo cual potencia de forma implícita las TIC.

La figura 3 refleja la frecuencia con la cual los estudiantes reciben sus actividades de enseñanza-aprendizaje de forma presencial con apoyo de las TIC.

Figura 3. Frecuencia de uso de las TIC en asignaciones dentro de clases

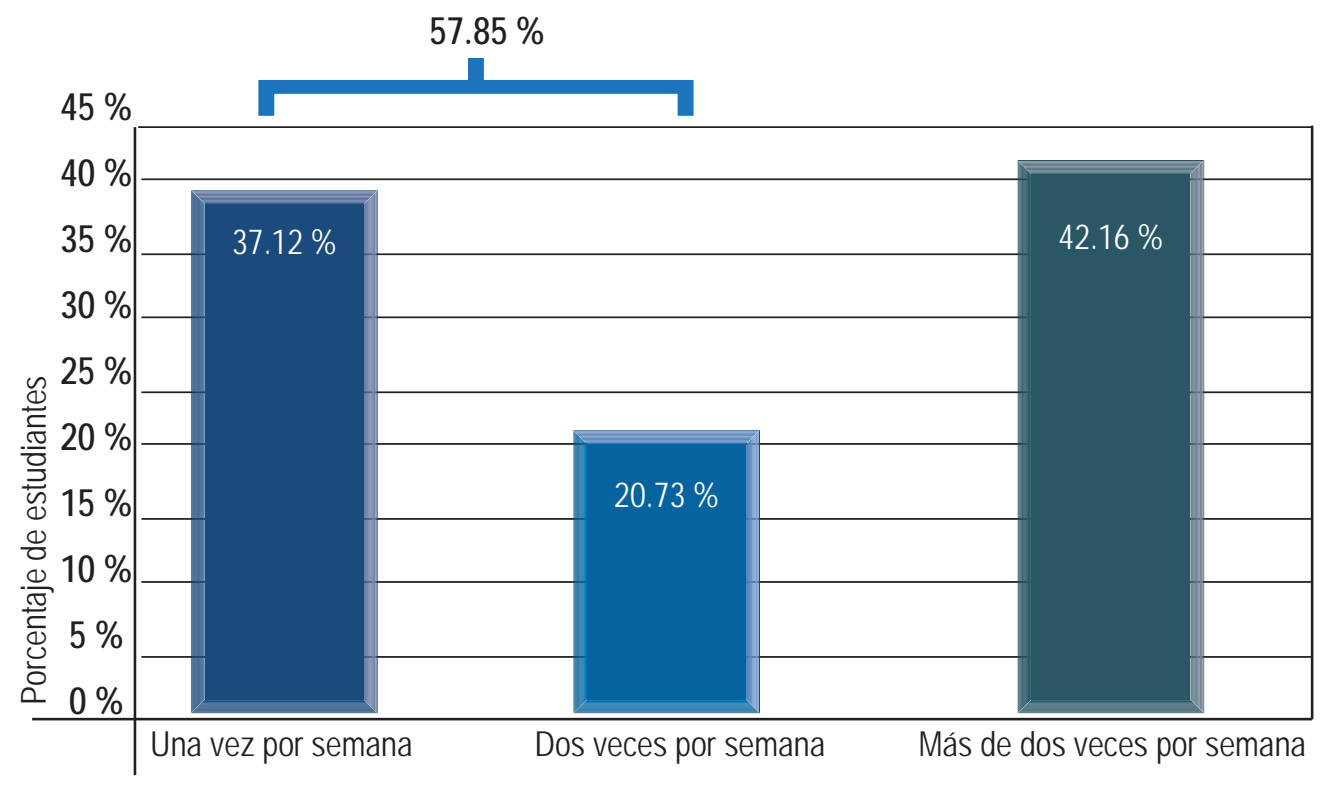

Asignaciones con TIC dentro del aula de clase

El uso de los recursos de parte del profesor dentro del aula de clase refleja un porcentaje bajo, sumando un $57.85 \%$ entre una y dos veces por semana, lo que se puede considerar preocupante, ya que debe existir una exigencia docente que permita la maximización del uso de las TIC en el aula y de todo el recurso que da la internet, sin olvidar el uso de las redes sociales y otras aplicaciones de intercambio de información existentes en el medio tecnológico. 


\section{Satisfacción estudiantil}

Para analizar la variable satisfacción estudiantil se definieron criterios como hardware (todo el equipo referente a las TIC), software (todo lo intangible del ambiente y que sirve de recurso tecnológico), condiciones de la internet (su ancho de banda y velocidades), el ambiente pedagógico de los laboratorios, el uso de la plataforma tecnológica (Moodle) y manuales de apoyo (orientado a mejorar el uso de los recursos).

Figura 4. Promedios de satisfacción, uso y aprovechamiento de las TIC por los estudiantes

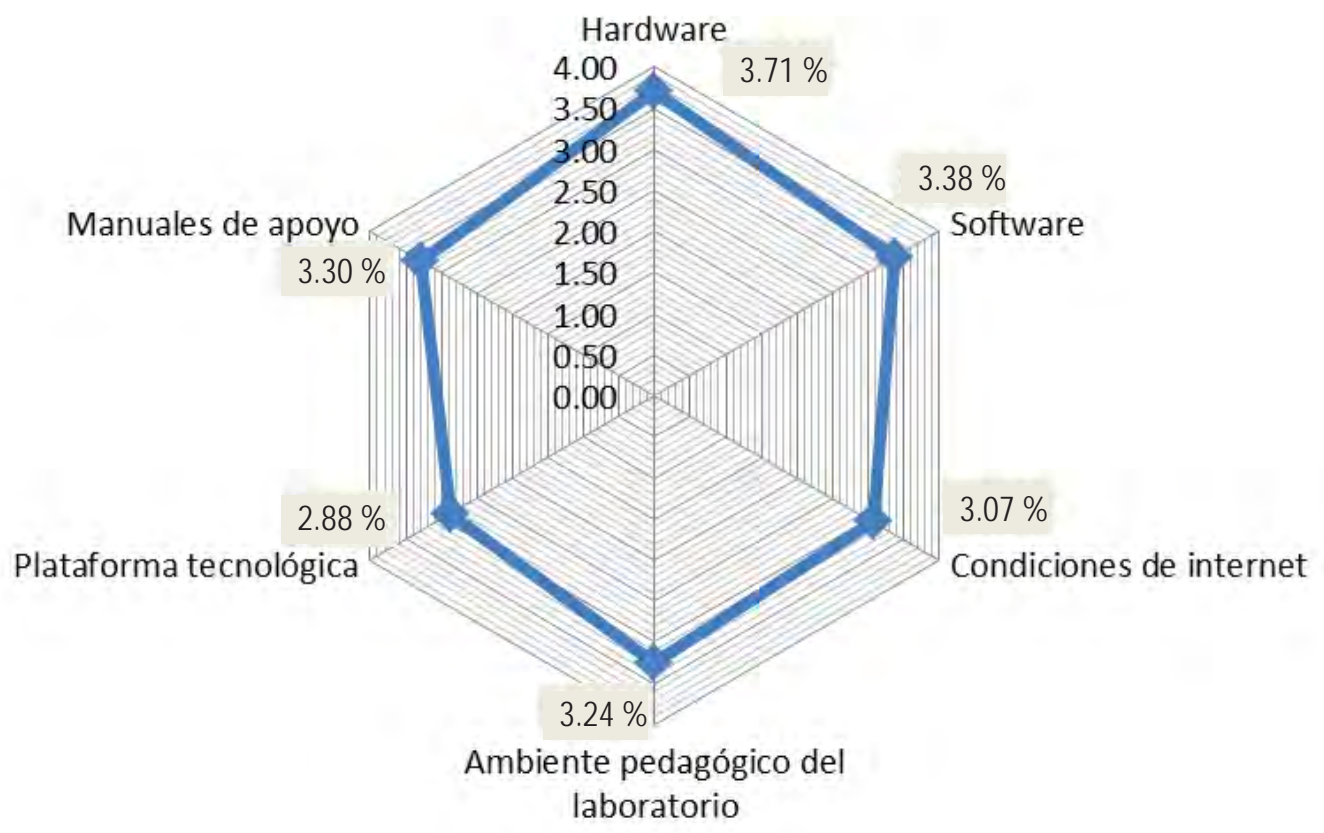

Los estudiantes afirman que dentro de la escala definida, el hardware y software suman muy buena satisfacción con relación a los demás elementos involucrados y a la vez vinculadas académicamente, reflejando un buen porcentaje de uso; pero aun en esta población muestra, se mantiene la percepción del bajo uso de la plataforma virtual Moodle y demás recursos virtuales proporcionados por ella (ver figura 4).

En cuanto al efecto de las TIC en el centro, se consideró en este caso su observación dentro y fuera del aula, obteniéndose datos sintetizados de las tres especialidades. 
Figura 5. Efecto del uso de las TIC en el contexto de la UNAH-TEC Danlí

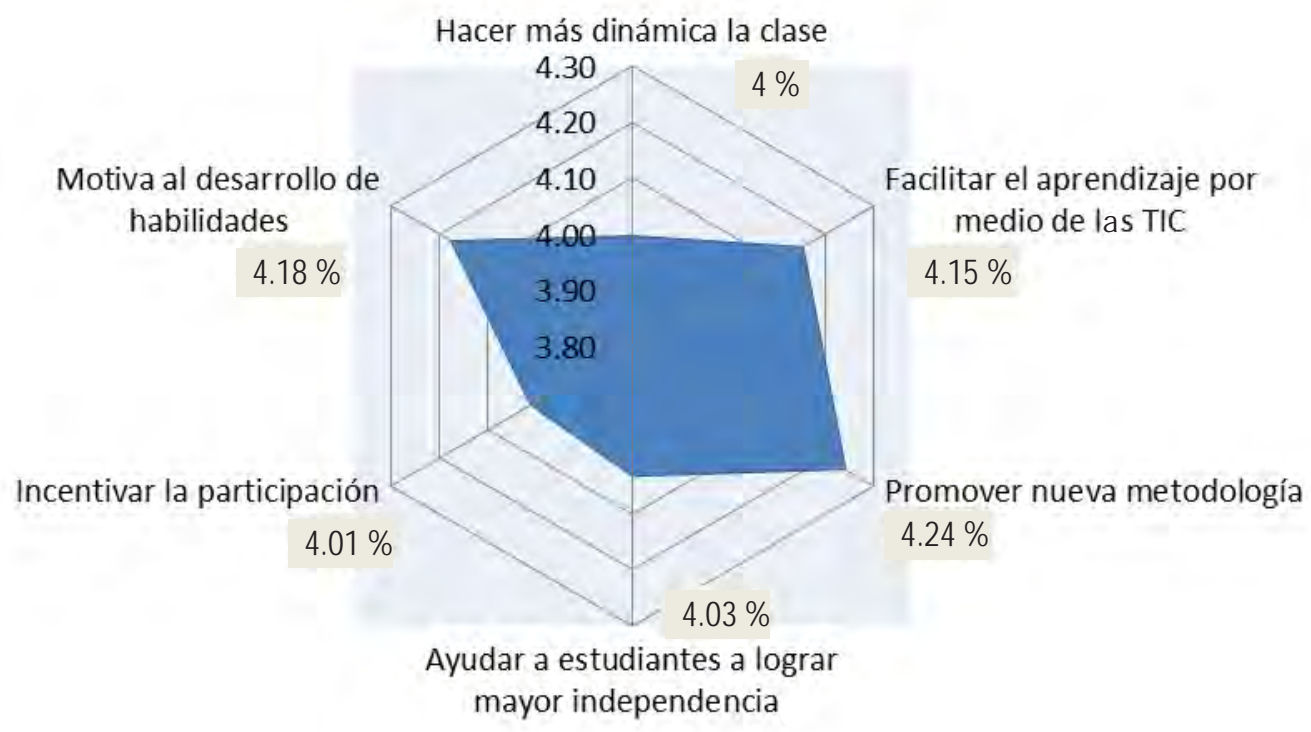

Se nota que la tecnología viene a promover una nueva metodología siendo, este el efecto más notorio, lo cual implica que nuestro ambiente académico es dinámico y flexible, tendiente a usar características de las teoría del conductismo y constructivismo, aunado al hecho de que el estudiante se siente más en un autoaprendizaje y logra más independencia como se observa en los resultados, sin olvidar el desarrollo de habilidades que se encuentran en un porcentaje alto.

Otro factor que suma a la satisfacción de los estudiantes con el uso de las TIC, es cómo se percibe el dominio de estas por parte de los profesores del centro. 
Figura 6. Satisfacción del dominio de las TIC en los profesores

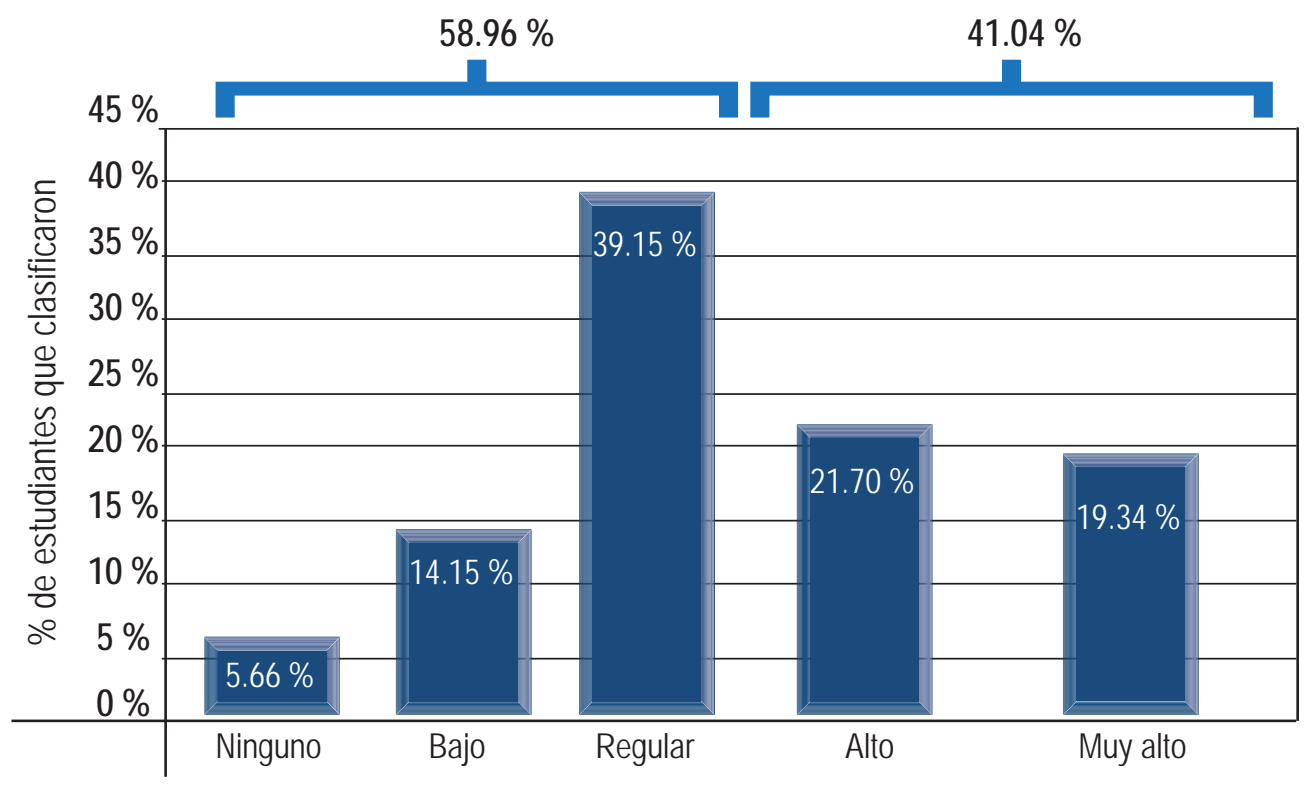

Nivel de dominio de TIC de docentes

El porcentaje que los estudiantes estiman sobre dominio de las TIC de parte de los docentes es un elemento a analizar y asumir en la gestión de cambio, notándose que al unir el criterio ninguno, bajo y regular, estos suman un $58.96 \%$, en contra del 41.04 $\%$ entre alto y muy alto en cuanto al dominio tecnológico, identificándose una debilidad en los profesores, los cuales deben preocuparse por mejorar la percepción que reflejan en el dominio de las TIC ante la población estudiantil.

\section{Rol del estudiante}

Para referirnos a este aspecto, se desarrolló una serie de interrogantes que buscaban evaluar cuál es el papel del alumno en el uso de las TIC en su ambiente universitario en la UNAH-TEC Danlí. 
Figura 7. Papel del alumno al usar las TIC en el proceso de enseñanza

Orientado al autoaprendizaje

Pasivo en el proceso

Activo en el proceso

Fomenta la investigación

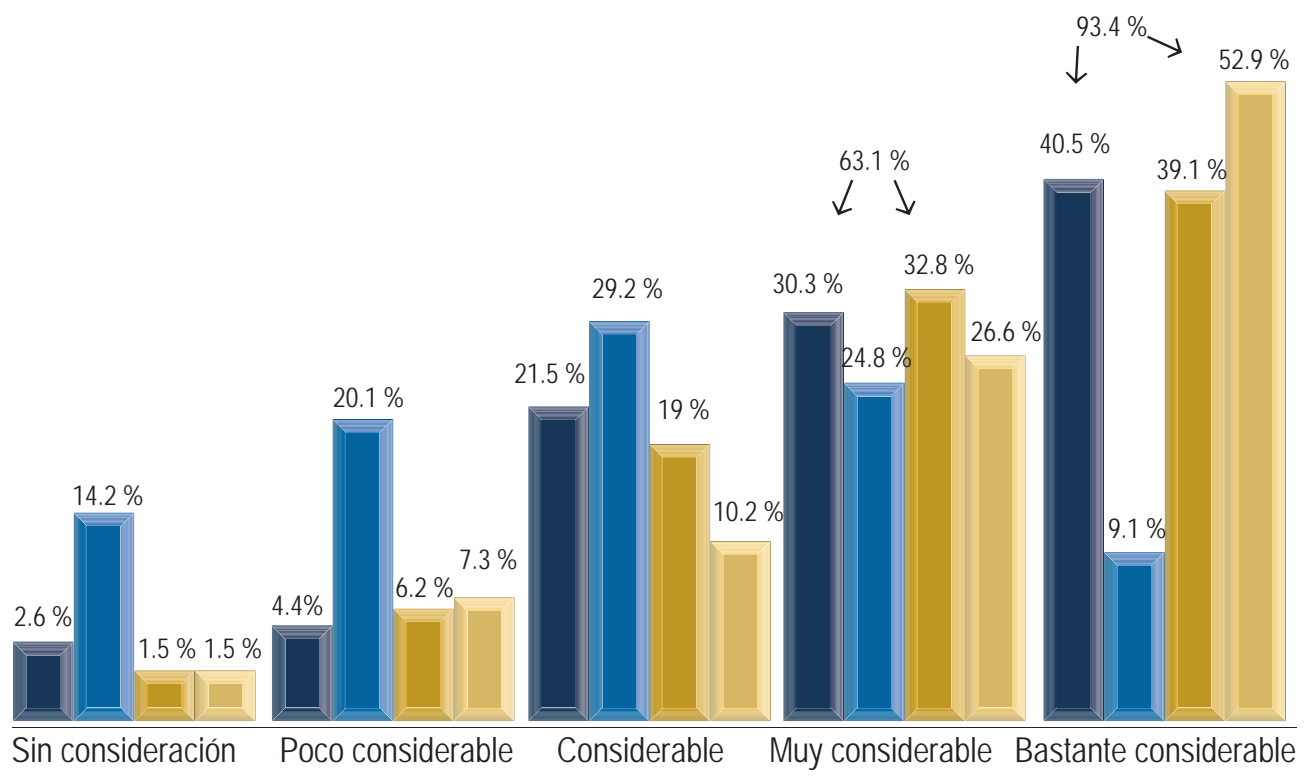

El grupo de estudiantes considera que el aspecto de investigación, junto con el autoaprendizaje, es cultivado en nuestro ambiente académico, lo cual coincide con las observaciones que el contexto académico UNAH-TEC se pone en práctica el conductismo y constructivismo, además, refleja al estudiante como un actor activo del proceso de aprendizaje.

Al conocer cuál es el porcentaje de uso potencial del internet en el ambiente universitario se vuelve necesario monitorear el uso de la plataforma Moodle. 
Figura 8. Ventajas del uso de la plataforma Moodle

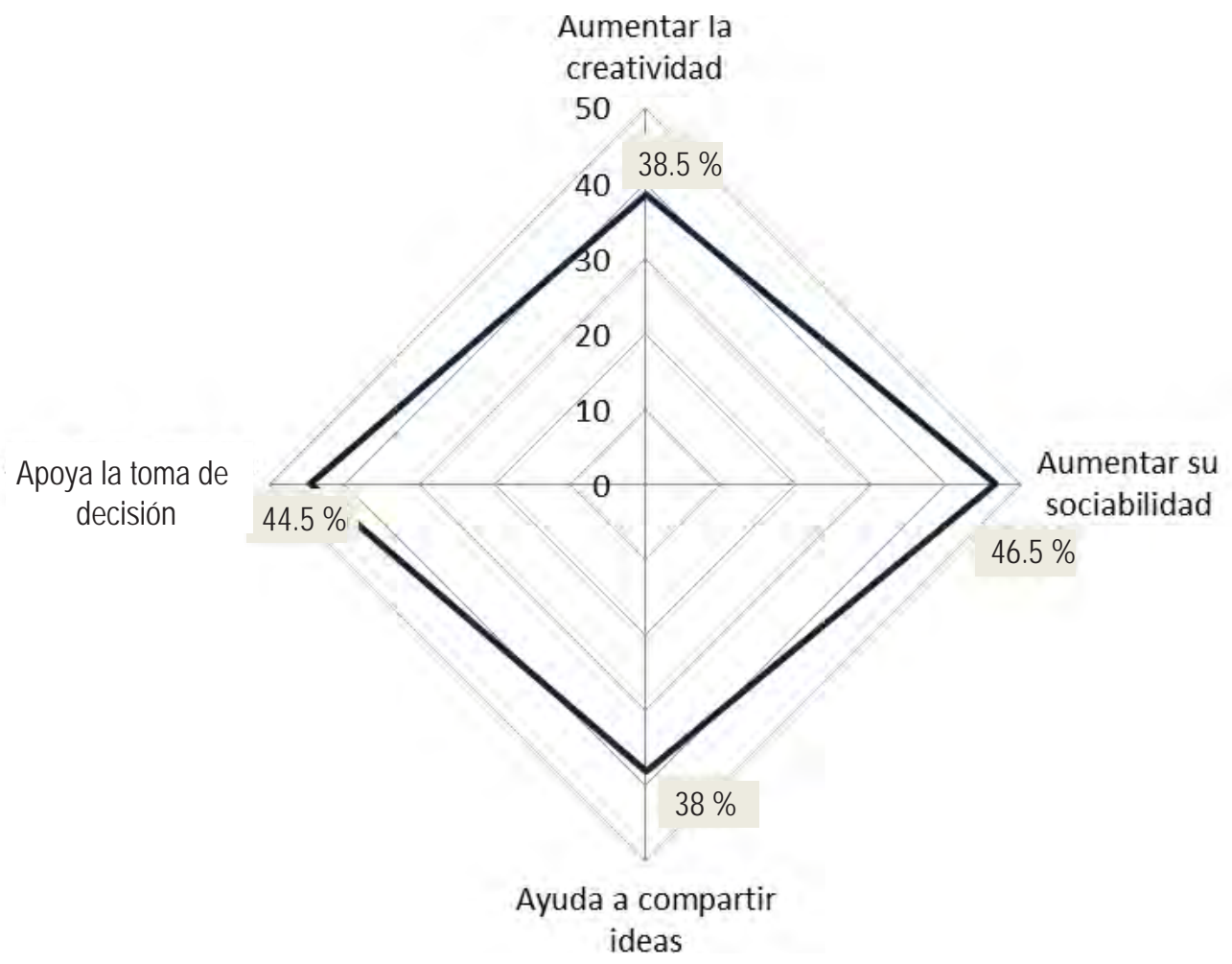

El aspecto tecnológico usado de forma tímida es la plataforma Moodle, en el cual la apreciación estudiantil se resume en que es una buena herramienta de apoyo al aumentar la sociabilidad y suma a la formación de un individuo, ideal para la toma de decisiones individuales y grupales, aumentando así la creatividad, aceptando que es un buen recurso de apoyo para compartir ideas.

\section{Rol del docente}

Uno de los factores que está ligado al manejo y uso de las TIC en los ambientes educativos son las teorías de aprendizaje y su aplicación en los aspectos metodológicos de enseñanza, volviéndose necesario captar la percepción de ellas en el contexto. 
Figura 9. Conocimiento y uso de las teorías de aprendizaje por parte del profesor

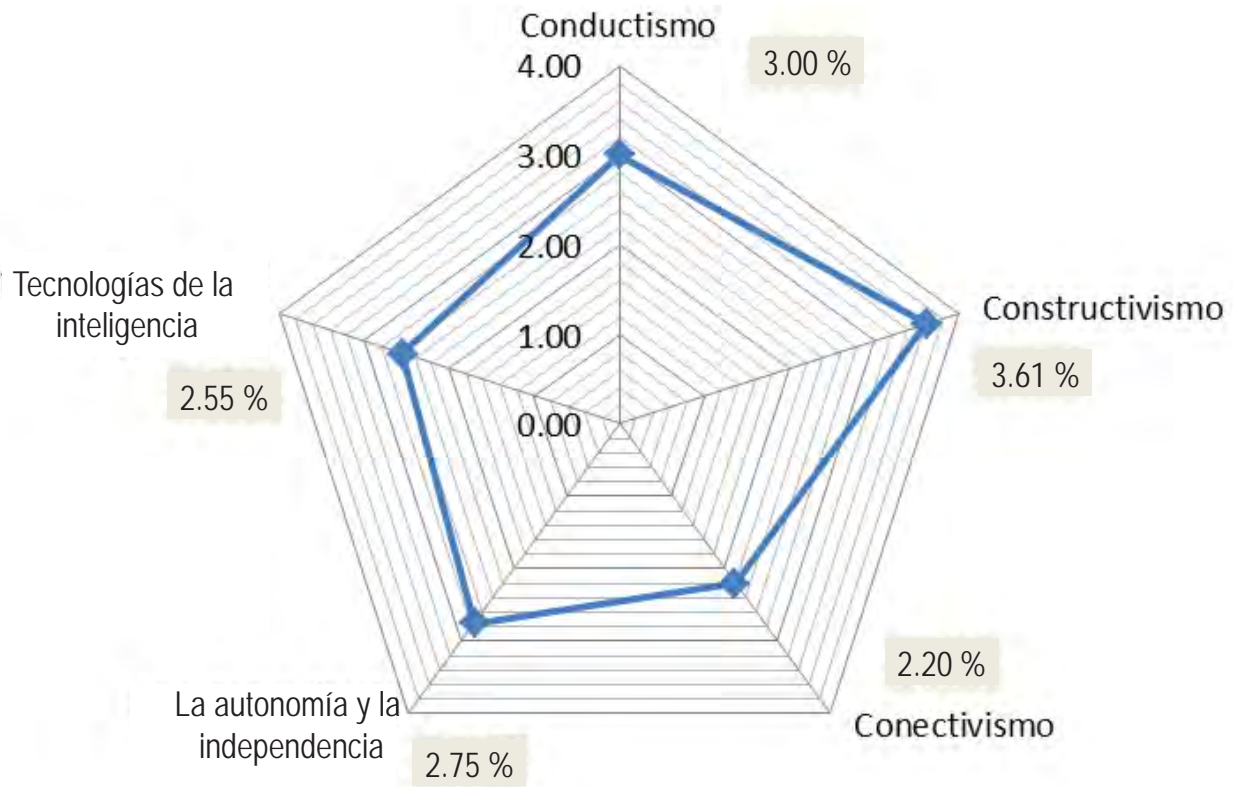

El profesor como elemento importante en el proceso académico en este contexto, hace uso de la teoría conductista y constructivista arriba del $3.0 \%$, pero refleja desconocimiento de las nuevas teorías emergentes referente a los procesos de cómo el individuo aprende, sumando un $2.55 \%$, observándose la necesidad de paliar esta limitante mediante capacitaciones, ya que es una exigencia a nivel superior que debe reflejarse en sus actividades a realizar en el aula mediante su planificación.

Ligado al conocimiento de las teorías de aprendizaje es necesario percibir el papel que cumple el profesor en el aula de clase con relación a su metodología. 
Figura 10. Papel del profesor en el proceso de enseñanza-aprendizaje

Orientado al proceso
Director del proceso
Asesor del proceso
Facilitador de proceso

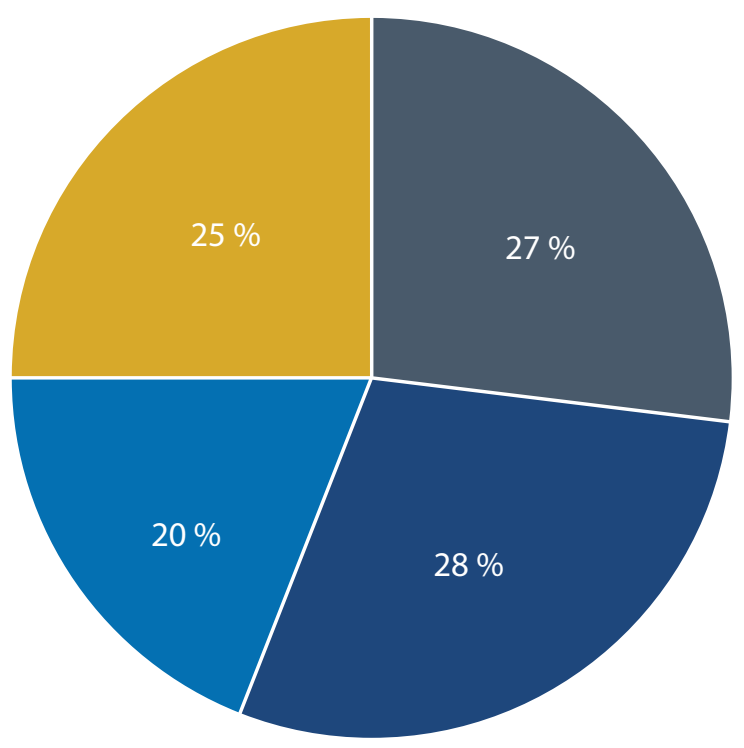

Los porcentajes permiten definir, a partir de las teorías de aprendizaje, que los profesores de la UNAH-TEC Danlí muestran una tendencia a la transición de una metodología de enseñanza conductista hacia una metodología constructivista, viéndose reflejado en las características de cómo ser orientador del proceso y facilitador, pero aun así se observa la necesidad de capacitación para profundizar en el uso y manejo de estas dos teorías y de las nuevas teorías del aprendizaje del conocimiento emergentes.

Las teorías y los papeles que los docentes desempeñan se encuentran ligados a un tercer factor, que es la intensidad en el uso de las TIC como recurso didáctico. 
Figura 11. Uso de los recursos tecnológicos existentes en el medio como recurso didáctico

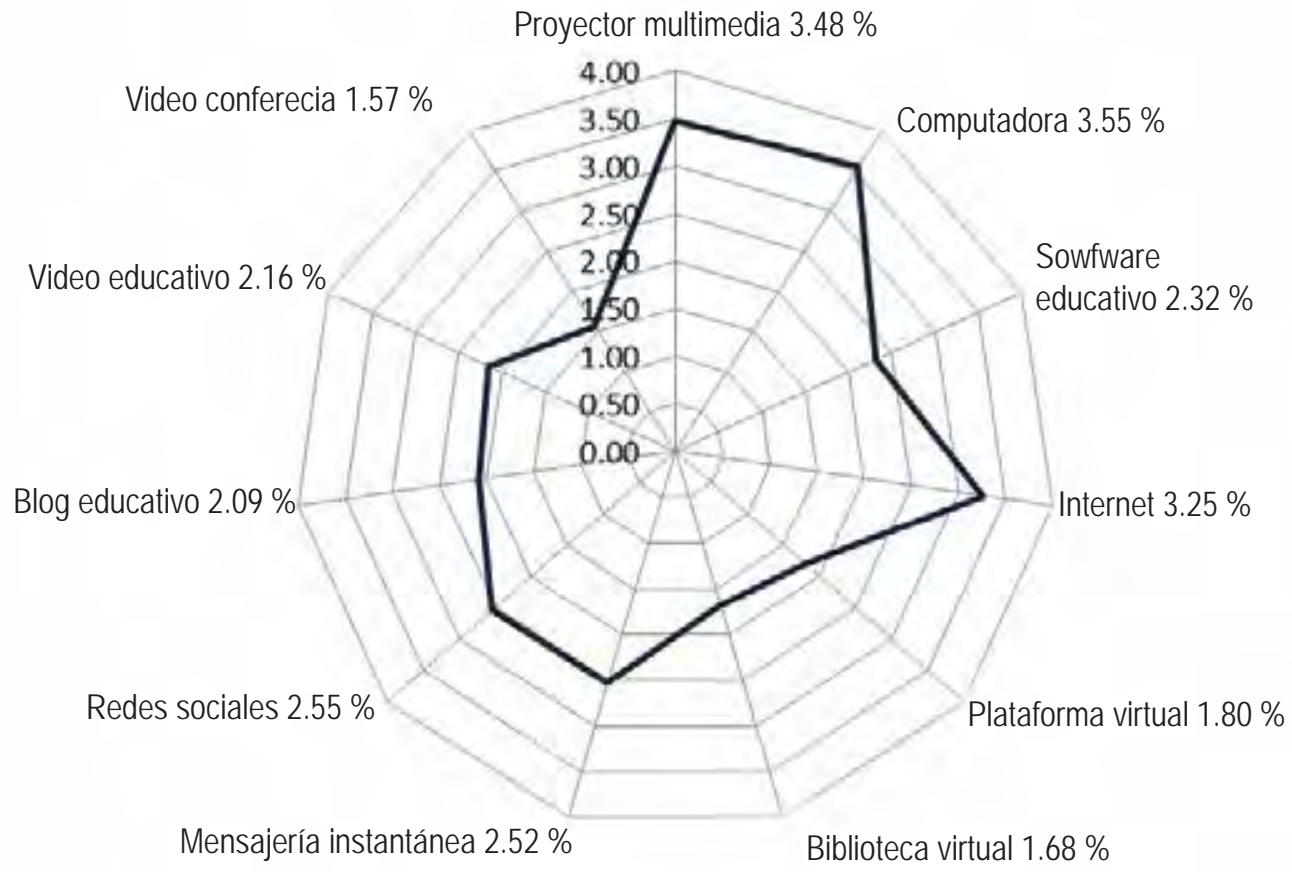

En el ambiente UNAH-TEC Danlí se puede observar que todos los recurso tecnológicos son usados de alguna manera, pero se visualiza que el proyector multimedia y la computadora tienen el mayor porcentaje ligado al uso del internet, pero se maximiza un uso en las redes sociales y sus aplicaciones para mensajerías instantáneas, dejando en un menor rango las videoconferencias y las bibliotecas virtuales ligadas al uso de la plataforma virtual Moodle.

\section{Formación y capacitación}

Para conocer la mejora en cuanto a la actualización del profesor con relación a contenidos en TIC, se considera en el diagnóstico la intensidad con la que ellos son capacitados por año. 
Figura 12. Intensidad de capacitaciones en TIC para profesores

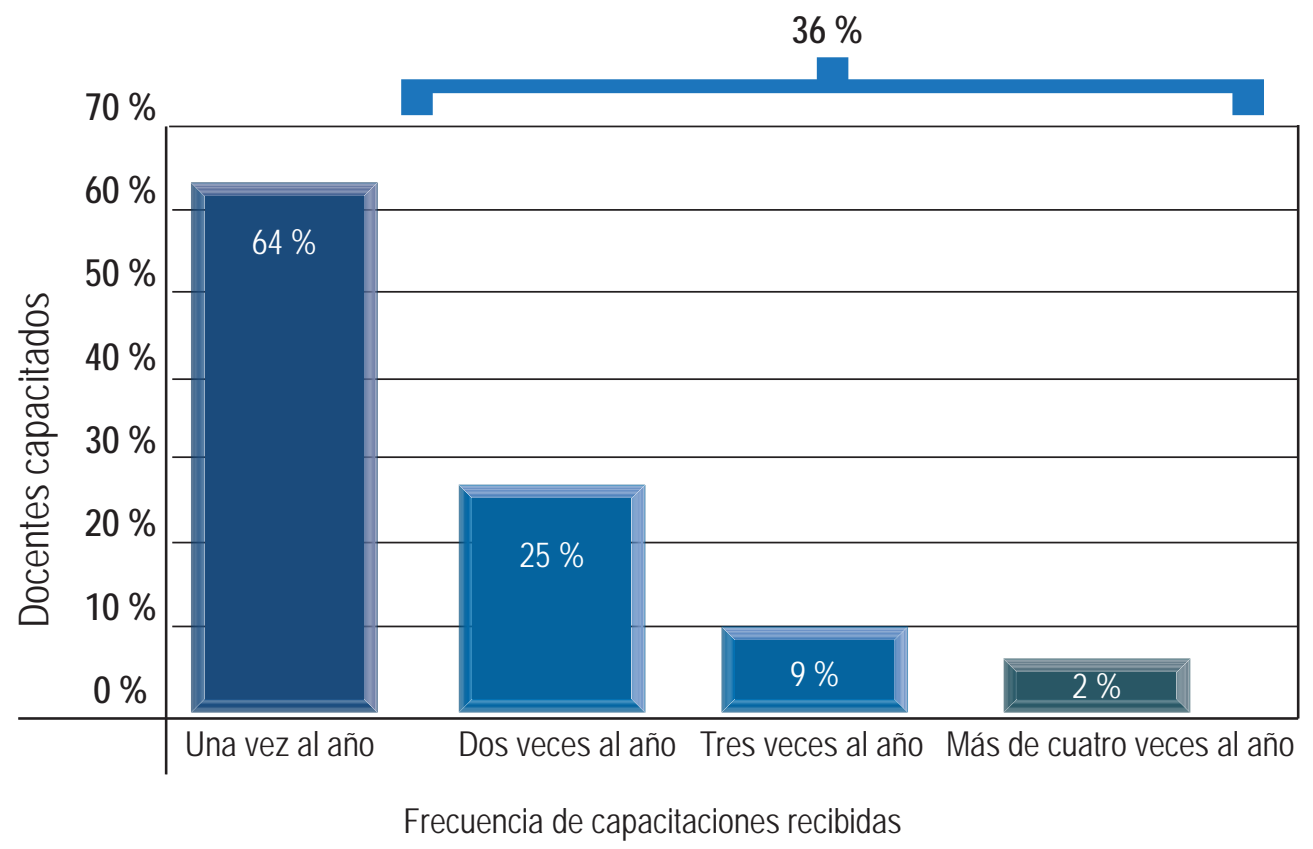

En cuanto a las capacitaciones docentes, este es uno de los elementos a los que se debe enfocar más la atención, ya que la mayoría solo se capacita una vez al año, observándose debilidad. Dado que existe diversidad de temas prioritarios, es de hacer notar que aun sumando los criterios dos, tres, cuatro veces al año, suman un $36 \%$, lo cual es extremadamente bajo.

Lo anterior está ligado a la priorización de temas para el grupo de profesores de la UNAH-TEC Danlí; se hizo una lista de temas de capacitación incluidos en la investigación que representan implícitamente el nivel y las etapas de implementación que las TIC conllevan. 
Figura 13. Prioridad de temas de capacitación para profesores
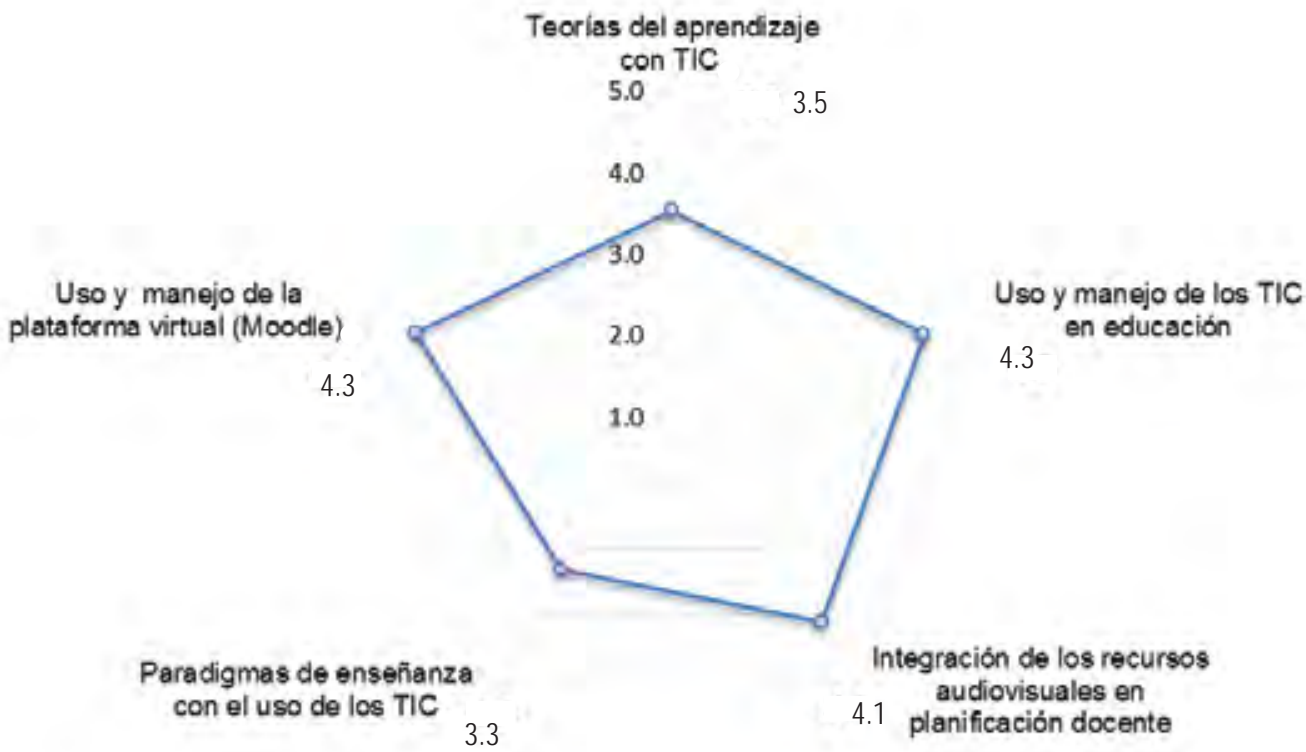

En las prioridades en temas de capacitación sobresalen el uso y manejo de las TIC en educación, seguida del uso y manejo de la plataforma Moodle; además de la integración de los recursos audiovisuales en la planificación docente, sin olvidar el fortalecimiento de las nuevas teorías de aprendizaje.

\section{Estado de los recursos tecnológicos existentes}

La descripción de variable estado del recurso de las TIC, en la investigación se apoya en la observación directa y en la verificación de la capacidad técnica computacional existente del hardware-software, permitiendo definir lo siguiente.

Los recursos tecnológicos existentes en UNAH-TEC, Danlí se encuentran en estado regular en cuanto a soporte técnico, teniendo actualmente su primer laboratorio de computación con capacidad para 30 estudiantes y 35 computadoras disponibles para su uso, con su respectivo aire acondicionado; también posee una pizarra electrónica digital, con proyector integrado, de larga duración en cuanto a su vida útil.

Este laboratorio refleja un ambiente pedagógico muy pobre, limitado por la falta de recursos económicos presupuestarios, lo que limita un mejor acondicionamiento del local; al igual, el mantenimiento óptimo de la computadoras es escaso, es por esta 
razón que algunos estudiantes se ven en la necesidad de portar su propia computadora personal para el desarrollo de la temática técnica de cada asignatura y demás proyectos tecnológicos.

Existe un segundo laboratorio de computación con 15 computadoras de escritorio, con el acondicionamiento de sillas y muebles ergonómicos con su correspondiente aire acondicionado; igualmente cuenta con conexión a internet, el cual es usado por estudiantes y profesores en actividades académicas de investigación, pero no se maximiza en el uso de la plataforma virtual, ya que el ancho de banda es muy limitado, está debajo de 2000 kbps de velocidad, lo que quiere decir que hay limitación en su conexión para la población estudiantil del centro universitario.

En cuanto al uso de la plataforma virtual, esta no es explotada con respecto a los servicios que presta y factores como el uso de bibliotecas virtuales, virtualización de asignaturas, organización de blog educativos, acceso y flexibilidad de las redes sociales, mensajerías instantáneas y la falta de un acceso a bases de datos internacionales.

Cada uno de los departamentos de la estructura organizativa posee proyectores multimedia, los cuales permiten a cada profesor hacer uso de ellos cuando la clase lo amerite de acuerdo al contenido temático. También, cada profesor lleva su computadora personal como valor agregado a la academia, permitiendo así poner en práctica su uso como recurso de apoyo al proceso de enseñanza-aprendizaje.

\section{CONCLUSIONES}

A continuación se presentan las conclusiones vinculadas a los resultados obtenidos, relacionados a las fuentes primarias y secundarias, proporcionan una visión más amplia de la solución al problema de investigación planteado.

1. En los recursos de las TIC disponibles, ya sean parte de la infraestructura de UNAH-TEC Danlí o como recurso facilitado por DEGT- UNAH, se identifica un nivel de intensidad de uso regular, tanto por profesores como por estudiantes.

2. La maximización del internet en el contexto educativo se interpreta como un medio desaprovechado, considerando los usos que permite, al ser una red abierta a servicios de comunicación. 
3. Los estudiantes y profesores muestran una baja satisfacción en el uso y aprovechamiento de las TIC de forma general, recalcando el uso de la plataforma Moodle, la cual indica un bajo uso.

4. Los estudiantes se identificaron con una actitud positiva, lo cual los coloca en un nivel con alto interés para incorporar el uso de las TIC en el proceso educativo, es de hacer notar que la tendencia es a pasar de una teoría conductista a una constructivista, variando de esta manera su rol actual dentro del proceso de enseñanza.

5. Se evidencia un alto interés del profesor en el uso del recurso tecnológico existente y, al mismo tiempo, en la investigación, uso pedagógico de las TIC y de las teorías de enseñanza- aprendizaje.

6. La mayoría de los profesores conocen y hacen uso de las teorías conductistas y constructivistas, reflejando conocimiento de ellas, pero dicen desconocer las nuevas teorías emergentes.

7. En factores de formación y capacitación, las TIC reflejan temas prioritarios como la planificación docente y manejo de la plataforma virtual Moodle, en su mayoría los profesores solo son capacitados una vez al año en temas que son de alto nivel de interés y prioridad para ellos.

8. Se observa que la infraestructura tecnológica disponible en la UNAH-TEC Danlí se encuentra en estado regular, con poca frecuencia de soporte técnico dirigido al mantenimiento de un estado óptimo funcional.

\section{RECOMENDACIONES}

1. Aplicar un plan de acción integrado de estratégicas que permitan la búsqueda de nuevas actividades didácticas haciendo uso de las TIC, fortaleciendo los recursos disponibles en el centro universitario.

2. Es necesario el mejoramiento de la conexión a internet como medio para potenciar los recursos de las TIC, mejorando y distribuyendo su ancho de banda de tal forma que no afecte el uso en los dos laboratorios computacionales. 
3. Gestionar en el plan de acción que potencie el uso y aprovechamiento de Moodle como plataforma virtual disponible en la UNAH-TEC Danlí, intensificando asignaciones virtuales.

4. Aprovechar la motivación y el alto grado de interés de los estudiantes en el uso e implementación de recursos tecnológicos, en las asignaciones dentro y fuera del salón de clase.

5. Es necesario ofrecer a los profesores la disponibilidad de recursos TIC, que permitan mantener en ellos una actitud de interés, impulsando la investigación y el uso en actividades didácticas.

6. Orientar y capacitar al profesor en el uso de metodologías que permita incluir las TIC como un recurso transversal e integrador de diferentes asignaturas.

7. Se recomienda la creación de un programa de formación en TIC para los docentes de la UNAH-TEC Danlí, con temas como el manejo de TIC en la educación, las TIC y la planificación docente y manejo de la plataforma virtual Moodle.

8. Definir planes de supervisión, monitoreo y evaluación del equipo tecnológico, incluyendo la propuesta de mantenimientos preventivos y correctivos necesarios, buscando un estado óptimo y funcional de la infraestructura tecnológica.

\section{AGRADECIMIENTOS}

A UNAH-TEC Danlí por haberme dado la oportunidad de ingresar al Sistema de Educación Superior y realizar esta investigación. Igualmente a los asesores temáticos y metodológicos por su apoyo, confianza y su capacidad para guiar el desarrollo de esta investigación. También a los catedráticos de la UNAH que facilitaron su tiempo en las diferentes asignaturas para la recopilación de la información, por su importante aporte y participación activa en la investigación, sumado a su disponibilidad y paciencia. Asimismo, a la Dirección de Investigación Científica y Posgrado de la UNAH, por su financiamiento para esta investigación. 


\section{BIBLIOGRAFÍA}

Arancibia, M. V. (2005). Muestreo estadistico, diseño y aplicaciones. Chile: Universitaria.

Díaz, A. M. (1996). El portafolio del docente. Honduras: Departamento de Desarrollo Rural del Zamorano.

Gómez, M. M. (2006). Introduccion a la metodología de la investigacion cientifica. Argentina: Brujas.

Juares, S. (2013). Uso de las TIC en la educación, América Latina y el Caribe. IESALC-UNESCO.

McDougall, D. S. (201). Cómo utilizar sofware educativo. España: Morata.

Sampieri, R. H. (2006). Metodología de la investigacion. México: McGraw Hill Interamericana.

Saravia, J. B. y Eguigure, Y. (2002). Informática educativa. Honduras: Guaymuras.

Soleno, R. D. y Morel, J. S. (2002). Gestión educativa institucional. Tegucigalpa: Ideas Litográficas.

Tamayo, M. T. (2001). El proceso de la investigacion cientifica. México: Limusa.

Suárez, E. J. (2008). El dashboard digital del docente. Chile: ELIZCOM. 